\title{
Vorwort zur Reihe
}

»Der Krieg ist nichts als die Fortsetzung der politischen Bestrebungen mit veränderten Mitteln. [...] Durch diesen Grundsatz wird die ganze Kriegsgeschichte verständlich, ohne ihn ist alles voll der größten Absurdität." Mit diesen Sätzen umriss Carl von Clausewitz im Jahre 1827 sein Verständnis vom Krieg als historischem Phänomen. Er wandte sich damit gegen die zu seiner Zeit und leider auch später weit verbreitete Auffassung, wonach die Geschichte der Kriege in erster Linie aus militärischen Operationen, aus Logistik, Gefechten und Schlachten, aus den Prinzipien von Strategie und Taktik bestünde. Für Clausewitz war Krieg hingegen immer und zu jeder Zeit ein Ausfluss der Politik, die ihn hervorbrachte. Krieg kann demnach nur aus den jeweiligen politischen Verhältnissen heraus verstanden werden, besitzt er doch allenfalls eine eigene Grammatik, niemals jedoch eine eigene Logik.

Dieser Einschätzung des Verhältnisses von Krieg und Politik fühlt sich Krieg in der Geschichte grundsätzlich verpflichtet. Die Herausgeber legen also Wert darauf, bei der Untersuchung der Geschichte der Kriege den Blickwinkel nicht durch eine sogenannte militärimmanente Betrachtungsweise verengen zu lassen. Doch hat seit den Zeiten Clausewitz' der Begriff des Politischen eine erhebliche Ausweitung erfahren. Die moderne Historiographie beschäftigt sich nicht mehr nur mit Außen- und mit Innenpolitik, sondern auch mit der Geschichte von Gesellschaft, Wirtschaft und Technik, mit Kultur- und Mentalitätsgeschichte und, nicht zuletzt, mit der Geschichte der Beziehungen zwischen den Geschlechtern. All die diesen unterschiedlichen Gebieten eigenen Aspekte haben die Geschichte der Kriege maßgeblich mitbestimmt. Die moderne historiographische Beschäftigung mit dem Phänomen Krieg kann deshalb nicht umhin, sich die methodologische Vielfalt der gegenwärtigen Geschichtswissenschaft zunutze zu machen. In diesem Sinne ist Krieg in der Geschichte offen für die unterschiedlichsten Ansätze in der Auseinandersetzung mit dem historischen Sujet.

Diese methodologische Offenheit bedeutet jedoch auch, dass Krieg im engeren Sinne nicht das alleinige Thema der Reihe sein kann. Die Vorbereitung und nachträgliche »Verarbeitung « von Kriegen gehören genauso dazu wie der gesamte Komplex von Militär und Gesellschaft. Von der Mentalitäts- und Kulturgeschichte militärischer Gewaltanwendung bis hin zur Alltagsgeschichte von Soldaten und Zivilpersonen sollen alle Bereiche einer modernen Militärgeschichte zu Wort kommen. Krieg in der Geschichte beinhaltet demnach auch Militär und Gesellschaft im Frieden.

Geschichte in unserem Verständnis umfasst den gesamten Bereich vergangener Realität, soweit sie sich mit den Mitteln der Geschichtswissenschaft erfassen läßt. In diesem Sinne ist Krieg in der Geschichte (abgekürzte Zitierweise: KRiG) grundsätzlich für Studien zu allen historischen Epochen offen, vom Altertum bis unmittelbar an den Rand der Gegenwart. Darüber hinaus ist Geschichte für uns nicht nur die vergangene Realität des sogenannten Abend- 
landes. Krieg in der Geschichte bezieht sich deshalb auf Vorgänge und Zusammenhänge in allen historischen Epochen und auf allen Kontinenten. In dieser methodologischen und thematischen Offenheit hoffen wir den spezifischen Charakter unserer Reihe zu gewinnen.

Stig Förster Bernhard R. Kroener Bernd Wegner Michael Werner 